\title{
Editorial: Data-Driven Energy Demand Side Management Techniques
}

\author{
Fengji Luo* \\ School of Civil Engineering, The University of Sydney, Darlington, NSW, Australia \\ (DD), energy consumption \\ Editorial on the Research Topic

\section{Data-Driven Energy Demand Side Management Techniques}

Keywords: demand response (DR), demand side management (DSM), smart grid (SG), energy systems, data driven

Ever-increasing prevalence of advanced metering infrastructure, Internet-of-Things (IoT) sensors, distributed energy resources and automation technologies have been significantly driving energy demand-side entities to operate from passive energy consumers to energy prosumers (producers-and-consumers) that proactively participate in the grid's operation. Further, these ubiquitous data acquisition facilities, together with recent machine learning advances, provide unprecedented opportunities to fuse multi-disciplinary knowledge to understand the energy customers' operational environments in a fine-grained manner and develop new data-driven demand side management techniques. Based on this background, this Special Article Collection is set up to solicit the latest and original contributions on datadriven demand side management techniques, five papers are collected in this special article collection, covering several important issues in data-driven demand side management techniques. Hirose studies the short- and medium-term electricity demand forecasting problem and proposes a statistical model that can effectively capture the nonlinear structure of the weather effect and interpret it into power load forecasting. Hirose validate the effectiveness of the technique in three real-world datasets. Zhao et al. propose an integrated smart appliance scheduling system that works on top of the home Internet-of-Things facilities. The system coordinately schedules different types of appliances in a smart home environment by considering the occupant's dwelling satisfaction. Li et al. develop a novel algorithm for demand side load event detection in smart homes. The algorithm is based on a wide-deep neural network that combines a convolutional neural network and a soft-max regressor; the deep model extracts the power time series patterns and the wide model utilizes the percentile information of the power time series. Wang et al. study the electricity retail pricing problem by considering the customer's demand response. They propose a coordinated optimization algorithm and a data-driven real-time pricing strategy in electricity markets. Ning et al. studies the power network reconfiguration problem by taking into account the distributed energy resources. They propose an edge-cloud coordinated reconfiguration framework with edge servers in the prosumers side and cloud servers in the utility grid side. In the framework, the edge computing technology are implemented in the edge servers to provide load forecasting, while cloud computing is used in the cloud servers to reconstruct the DN.

We hope the papers collected in this special article collection can provide useful references to researchers and engineers and can advance the knowledge in energy demand response, demand side management, and smart grids. 


\section{AUTHOR CONTRIBUTIONS}

FL is the sole author who writes the editorial of the special article collection.

Conflict of Interest: The author declares that the research was conducted in the absence of any commercial or financial relationships that could be construed as a potential conflict of interest.

Publisher's Note: All claims expressed in this article are solely those of the authors and do not necessarily represent those of their affiliated organizations, or those of the publisher, the editors and the reviewers. Any product that may be evaluated in this article, or claim that may be made by its manufacturer, is not guaranteed or endorsed by the publisher.

Copyright (C) 2022 Luo. This is an open-access article distributed under the terms of the Creative Commons Attribution License (CC BY). The use, distribution or reproduction in other forums is permitted, provided the original author(s) and the copyright owner(s) are credited and that the original publication in this journal is cited, in accordance with accepted academic practice. No use, distribution or reproduction is permitted which does not comply with these terms. 